\title{
Size Matters: Why Optic Disk Size should be Measured When Assessed for Glaucoma?
}

\author{
${ }^{1}$ Yaniv Barkana, ${ }^{2}$ Robert Ritch \\ ${ }^{1}$ Glaucoma Service, Department of Ophthalmology, The Assaf Harofe Medical Center in Zerifin, Israel \\ e-mail: yaniv.barkana@ yahoo.com \\ ${ }^{2}$ Glaucoma Service, Department of Ophthalmology, The New York Eye and Ear Infirmary, New York
}

\section{INTRODUCTION}

Examining the optic disk is crucial to detecting glaucoma. Correct diagnosis requires measuring the disk's size in particular. Knowledge of the disk size is very important for an accurate diagnosis of glaucoma. Same cup size in two different disks may have different implications. Disk size can be measured clinically as well as with optic nerve imaging devices, HRT, OCT, GDx. In all glaucoma workups, size of disk should be mentioned as average normal, smaller or large.

\section{WHY MEASURE THE OPTIC DISK?}

The answer is based on two fundamental structural observations: First, the optic disk area varies widely among individuals by a factor of approximately 1:7. ${ }^{1}$ The horizontal and vertical disk diameters are also highly variable, although to a somewhat lesser extent. ${ }^{2}$ Second, the size of parameters such as the neuroretinal rim, the cup, and the commonly used cupto-disk ratio are determined by the optic disk's size. ${ }^{3-5}$ Thus, a large cup-to-disk ratio may be physiologic if the optic disk is large, ${ }^{6}$ whereas even a small cup-to-disk ratio may signify glaucomatous optic neuropathy in the presence of a small disk. ${ }^{7}$ Therefore, when evaluating an optic disk such as the one shown in Figure 1 it is essential to know its size. Moreover, recent systems for staging glaucomatous optic disk damage require the consideration of disk size. ${ }^{8}$ One also might view differently the results of population-based glaucoma prevalence studies in which an arbitrary cup-to-disk ratio was used as a diagnostic criterion without accounting for disk size. This is analogous to current interpretation of previous studies that reported IOP in large populations without taking accounting for corneal thickness. Moreover, asymmetric cupping, commonly interpreted as a sign signifying possible glaucoma, may indeed be a physiologic result of asymmetric disk size rather than an indication of glaucoma (Abstract, Bujak and Yan, ARVO 2004).

The Moorfields Regression Analysis used by the Heidelberg Retina Tomograph (HRT; Heidelberg Engineering $\mathrm{GmbH}$, Dossenheim, Germany) takes disk area into account, because it was shown to improve discrimination between healthy disks and those with early glaucoma. ${ }^{9}$ Investigators in the scanning laser ophthalmoscopy ancillary study to the Ocular Hypertension Treatment Study found that most optic disk parameters were significantly larger in blacks than in other racial groups. When disk size was included in the multivariate analysis, however, these differences were no longer statistically significant. ${ }^{10}$ The investigators suggested that the size of patients' optic disks must be considered in addition to their race when diagnosing glaucoma (Fig. 1).

In the standard printout of the HRT3 (Heidelberg Engineering $\mathrm{GmbH}$ ), the statistical normality analysis of every disk parameter is adjusted for the disk's size. Researchers have also recently reported correlating the size of the optic disk to measurements of the retinal nerve fiber layer's thickness by optical coherence tomography (OCT $)^{11}$ and scanning laser polarimetry. ${ }^{12}$

\section{HOW TO MEASURE THE OPTIC DISK?}

The clinician may measure the vertical and horizontal disk diameters at the slit lamp using contact or noncontact lenses. A narrow beam is projected onto the disk, its length is adjusted to the diameter of the disk within (but not including) the peripapillary scleral ring, and this length is read on the scale of the slit-lamp in millimeters. ${ }^{13-15}$ The clinician may hold the lens with his thumb and index finger while his fourth finger rests on the patient's cheekbone; this approach keeps a constant length between the lens and the patient's eye, and it also maintains the stability of the lens, thus allowing more precise measurements. Different fundus lenses provide different magnification of fundus objects. ${ }^{15,16}$ Thus, in order to obtain the actual disk size, the magnification specific to the lens used must be known. However, as explained below, this may not be necessary in routine clinical measurement. The eye's refraction, when not unusually ametropic, and the distance between the fundus lens and eye, do not significantly alter the measurement. ${ }^{16}$

Novel imaging devices such as the HRT and Stratus OCT (Carl Zeiss Meditec, Inc., Dublin, CA) analyze the optic disk, 
Fig. 1: This patient has IOPs under $20 \mathrm{~mm} \mathrm{Hg}$, full visual fields, and optic disks that are suspicious for glaucoma. The clinician must measure the size of these disks before making a diagnosis, however

including its size. The standard OCT printout includes a measurement of the disk area and vertical diameter. The standard HRT printout includes disk area, and the clinician may request the calculation of any disk diameter via the "interactive measurements" option. The HRT is dependant on the subjective outlining of the nerve by the examiner. The OCT can either automatically determine disk size by detecting the edge of the RPE, or this can be done manually by the examiner; these two methods have been found to differ significantly in many cases. ${ }^{17}$ These automated or semi-automated measurements have higher reproducibility and lower interobserver variability compared with the clinician's own assessment of the optic disk. In addition, the printouts allow easy documentation and archiving of the disk's status. For these reasons, practitioners are relying increasingly on these instruments as diagnostic tools, even to the point of replacing a careful funduscopic evaluation.

\section{ARE DISK SIZE MEASUREMENTS BY DIFFERENT METHODS INTERCHANGEABLE?}

At present, the disturbing answer is-no. The range of differences in measurements of the size and other parameters of the optic disk between various modalities does not allow their interchangeability or the provision of conversion formulas. ${ }^{17-}$

19 Realizing this, Jonas suggested that, in daily practice, clinicians need not incorporate into their evaluations the exact numerical value of the disk's size and various conversion formulae. ${ }^{14}$ Instead, he recommended using a quick, crude estimate of whether the disk in question was of average, smaller, or larger size. Even so, in a recent study, we found poor agreement between the HRT-II (Heidelberg Engineering GmbH), the Stratus OCT, and funduscopy-both in absolute measurements of the optic disk's size, and in the categorization of disks as small, average, or large (Fig. 2). ${ }^{20}$

\section{PRACTICAL IMPLICATIONS}

Until further technological adjustments are made, the estimation of both the absolute and relative size of a disk can only be defined separately for each measuring technique. Because all of the modalities provide relatively reproducible measurements, however, they may each be used successfully to define a nomogram and thus for diagnostic purposes. Measuring the 

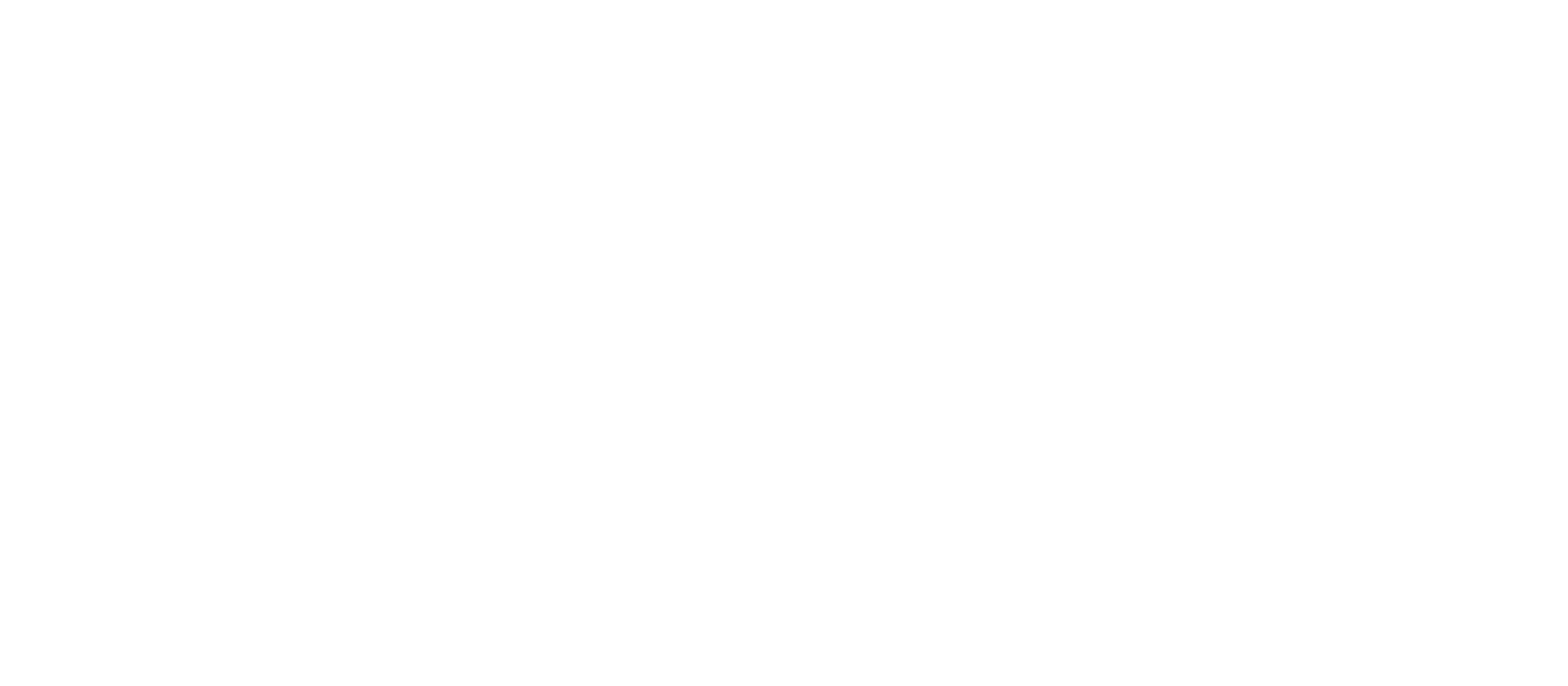

Fig. 2: The right and left disks of a 56 -year-old nurse who is followed as a glaucoma suspect. The left disk has a noticeably larger cup compared with the right disk, which barely has a cup at all. However, this may be a physiologic consequence of the fact that the left disk is larger-its vertical diameter measured at the slit lamp with a $60 \mathrm{D}$ lens is $1.8 \mathrm{~mm}$, whereas the right disk measures only $1.5 \mathrm{~mm}$

vertical or horizontal diameter of the optic disk at the slit lamp is the easiest and least time-consuming approach. One may use either diameter to classify the disk's size. For research purposes, one may use both diameters in the following formula to calculate: Disk area: area $=\pi \mathrm{r} / 4$ horizontal diameter $\times$ vertical diameter. $^{21}$

Measurements of the disk at the slit lamp are affected by magnification factors related to the slit lamp and lens used. Some standardization may be achieved by multiplying measured size by the magnification factor provided by the manufacturer for each lens. Alternatively, each clinician therefore should establish his own normal values, for example, by measuring the disks of 30 or so patients of the same ethnic background. During subsequent routine clinical examination, this allows the quick classification of the disk as average-sized, small or large, with immediate implications on the meaning of the observed disk parameters such as the cup, rim, and cup-disk ratio. Using machines such as the HRT provides a greater degree of standardization. For the classification of disk size, the clinician can grossly rely on the normal values provided by the instrument's database, provided he/she is familiar with the source of the database (e.g. race and age of the population) and its relevance to his own patients. For example, the HRT3 currently has ethnic-selectable databases, with the Caucasian database including some 700 healthy eyes. It classifies each optic disk as small, average, or large based on disk area (Fig. 3), with average disk area defined as mean $\pm 1 \mathrm{SD}\left(2.03 \pm 0.4 \mathrm{~mm}^{2}\right.$ for Whites).

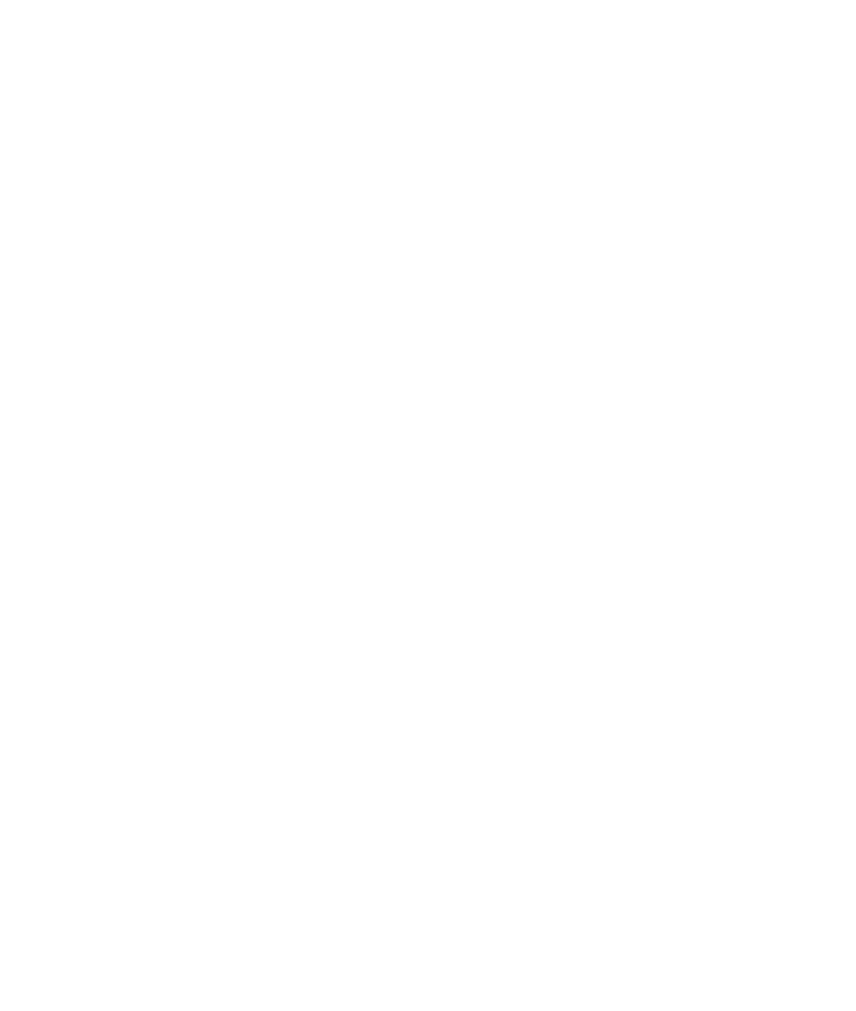

Fig. 3: This sample printout for the HRT3 demonstrates the importance of the optic disk's size to glaucoma. Disk area is categorized as small, average, or large based on an ethnic-specific database (Caucasian in this case). The statistical normality analysis of every disk parameter ( $P$ values) is adjusted for disk size 


\section{CONCLUSION}

Measuring the size of the optic disk is required for the accurate diagnosis of glaucoma. Without this information, clinicians may overdiagnose the disease in eyes with large disks and physiologic cupping, and they may miss early glaucoma in eyes with small disks and small cups. Whether measuring the disk at the slit lamp or with imaging devices such as the HRT or Stratus OCT, practitioners must be familiar with the methodology and variability of each method. At present, measurements are not interchangeable between these modalities.

\section{REFERENCES}

1. Jonas JB, Budde WM, Panda-Jonas S. Ophthalmoscopic evaluation of the optic nerve head. Surv Ophthalmol 1999;43:293320.

2. Crowston JG, Hopley CR, Healey PR, et al. The effect of optic disc diameter on vertical cup to disc ratio percentiles in a population based cohort: the Blue Mountains Eye Study. Br J Ophthalmol 2004;88:766-70.

3. Jonas JB, Gusek GC, Naumann GO. Optic disc, cup and neuroretinal rim size, configuration and correlations in normal eyes. Invest Ophthalmol Vis Sci 1988;29:1151-8.

4. Caprioli J, Miller JM. Optic disc rim area is related to disc size in normal subjects. Arch Ophthalmol 1987;105:1683-85.

5. Vernon SA, Hawker MJ, Ainsworth G, et al. Laser scanning tomography of the optic nerve head in a normal elderly population: the Bridlington eye assessment project. Invest Ophthalmol Vis Sci 2005;46:2823-8.

6. Jonas JB, Zach FM, Gusek GC, Naumann GO. Pseudoglaucomatous physiologic large cups. Am J Ophthalmol 1989;107:13744.

7. Jonas JB, Fernandez MC, Naumann GO. Glaucomatous optic nerve atrophy in small discs with low cup-to-disc ratios. Ophthalmology 1990;97:1211-5.

8. Spaeth GL, Lopes JF, Junk AK, Grigorian AP, Henderer J.Systems for staging the amount of optic nerve damage in glaucoma: a critical review and new material. Surv Ophthalmol 2006;51:293-315.

9. Wollstein G, Garway-Heath DF, Hitchings RA. Identification of early glaucoma cases with the scanning laser ophthalmoscope. Ophthalmology 1998;105:1557-63.
10. Zangwill LM, Weinreb RN, Berry CC, et al. Racial differences in optic disc topography: baseline results from the confocal scanning laser ophthalmoscopy ancillary study to the ocular hypertension treatment study. Arch Ophthalmol 2004;122:228.

11. Savini G, Zanini M, Carelli V, Sadun AA, Ross-Cisneros FN, Barboni P. Correlation between retinal nerve fibre layer thickness and optic nerve head size: an optical coherence tomography study. Br J Ophthalmol 2005;89:489-92.

12. Laemmer R, Horn FK, Viestenz A, Juenemann AG, Mardin CY.Influence of optic disc size on parameters of retinal nerve fiber analysis with laser scanning polarimetry. Graefes Arch Clin Exp Ophthalmol 2006;244:603-8.

13. Ruben S. Estimation of optic disc size using indirect biomicroscopy. Br J Ophthalmol 1994;78:363-4.

14. Jonas JB, Papastathopoulos K. Ophthalmoscopic measurement of the optic disc. Ophthalmology 1995;102:1102-6.

15. Lim CS, O’Brien C, Bolton NM. A simple clinical method to measure the optic disc size in glaucoma. J Glaucoma 1996;5:2415.

16. Ansari-Shahrezaei S, Maar N, Biowski R, Stur M.Biomicroscopic measurement of the optic disc with a high-power positive lens. Invest Ophthalmol Vis Sci 2001;42:153-7.

17. Iliev ME, Meyenberg A, Garweg JG. Morphometric assessment of normal, suspect and glaucomatous optic discs with Stratus OCT and HRT II. Eye. September 23, 2005 [epub ahead of print].

18. Hoffmann EM, Bowd C, Medeiros FA, et al. Agreement among 3 optical imaging methods for the assessment of optic disc topography. Ophthalmology 2005;112:2149-56.

19. Jayasundera T, Danesh-Meyer HV, Donaldson M, Gamble G. Agreement between stereoscopic photographs, clinical assessment, Heidelberg retina tomograph and digital stereoscopic optic disc camera in estimating vertical cup:disc ratio. Clin Exp Ophthalmol 2005;33:259-63.

20. Barkana Y, Harizman N, Gerber Y, Liebmann JM, Ritch R. Measurements of optic disc size with HRT II, Stratus OCT and funduscopy are not interchangeable. Am J Ophthalmol 2006;142:375-80.

21. Jonas JB, Montgomery DM. Determination of the neuroretinal rim area using the horizontal and vertical disc and cup diameters. Graefes Arch Clin Exp Ophthalmol 1995;233:690-3. 